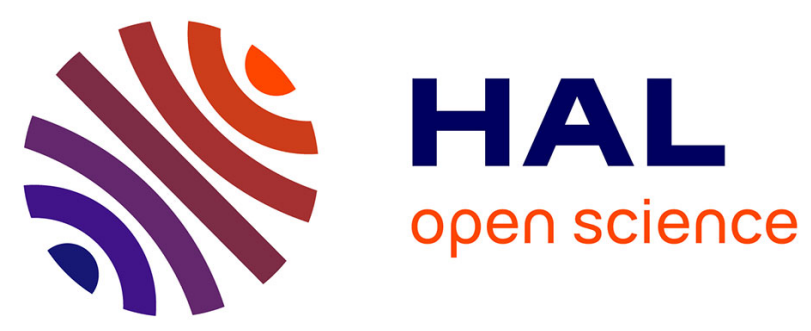

\title{
Approche psychomotrice de l'Être sous contrainte au cours des 24 premières heures d'hospitalisation en UNV-A après un AVC ischémique
}

Marie Agostinucci, Chantal Dutems-Carpentier, Sylvain Hanneton, Bernard Andrieu

\section{To cite this version:}

Marie Agostinucci, Chantal Dutems-Carpentier, Sylvain Hanneton, Bernard Andrieu. Approche psychomotrice de l'Être sous contrainte au cours des 24 premières heures d'hospitalisation en UNV-A après un AVC ischémique. L'Évolution Psychiatrique, 2019, 84 (2), pp.315-322. 10.1016/j.evopsy.2019.03.004 . hal-02955738

\section{HAL Id: hal-02955738 \\ https://hal.science/hal-02955738}

Submitted on 2 Oct 2020

HAL is a multi-disciplinary open access archive for the deposit and dissemination of scientific research documents, whether they are published or not. The documents may come from teaching and research institutions in France or abroad, or from public or private research centers.
L'archive ouverte pluridisciplinaire HAL, est destinée au dépôt et à la diffusion de documents scientifiques de niveau recherche, publiés ou non, émanant des établissements d'enseignement et de recherche français ou étrangers, des laboratoires publics ou privés. 


\section{Approche psychomotrice de l'Etre sous contrainte au cours des $\mathbf{2 4}$ premières heures d'hospitalisation en UNV-A après un AVC ischémique \\ Marie AGOSTINUCCI ${ }^{1}$, Chantal DUTEMS-CARPENTIER ${ }^{2}$, Sylvain HANNETON ${ }^{3}$, et Bernard ANDRIEU ${ }^{4}$}

${ }^{1}$ Marie AGOSTINUCCI, doctorante de l'EA3625 et psychomotricienne D.E du Centre d'Accueil de jour pour adultes cérébrolésés La Note Bleue de la Fondation Partage et Vie

${ }^{2}$ Chantal DUTEMS-CARPENTIER, psychomotricienne D.E de l'UNV-A de l'Hôpital Henri Mondor

${ }^{3}$ Sylvain HANNETON, directeur adjoint de l'EA 3625 L'institut des Sciences du Sport Santé I3SP

${ }^{4}$ Bernard ANDRIEU, directeur de l'EA 3625 L'institut des Sciences du Sport Santé I3SP

Agostinucci, M., Dutems-Carpentier, C., Hanneton, S., \& Andrieu, B. (2019). Approche psychomotrice de l'Etre sous contrainte au cours des 24 premières heures d'hospitalisation en UNV-A après un AVC ischémique. L'Évolution Psychiatrique, 84(2).

\section{Résumé}

Objectif - L'AVC constitue à la fois un enjeux majeur de santé publique et une urgence médicale. La prise en soin des patients dès les premières heures qui suivent l'AVC constitue une expérience déstructurante de l'Etre au monde pourtant essentielle à la survie.

Méthode - Une lecture phénoménologique des troubles sensorimoteurs et des contraintes médicales qui les accompagnent est proposée. A travers des illustrations cliniques au sein d'une UNV-A, l'approche psychomotrice est présentée.

Résultats - La posture et les soins apportés par la psychomotricienne sont orientés vers une activation des systèmes sensorimoteurs au seins d'une dynamique relationnelle, étayée par un apport de sens des éléments de contrainte.

Discussion -La psychomotricité permet ainsi de pallier certains effets délétères induits par les contraintes médicales par une expérience corporelle en relation. Tandis que l'approche psychomotrice de la contrainte, par l'explication au patient, rend acceptable le raccordement aux machines.

Mots clés : AVC ; Phénoménologie ; Psychomotricité ; Corps ; Contrainte

\section{Abstract}

Objective - Stroke is a major public health issue and a medical emergency. Caring for patients in the first few hours after a stroke is a destructive experience of Being in the world that is essential for survival.

Method - A phenomenological reading of sensorimotor disorders and the medical constraints that accompany them is proposed. Through clinical illustrations within a UNV-A, the psychomotor approach is presented.

Results - The posture and care provided by the psychomotor therapist are oriented towards the activation of sensorimotor systems within a relational dynamic, supported by an explanation of the elements of constraint. 
Discussion - Psychomotricity compensates some harmful effects induced by medical constraints through a related body experience. While the psychomotor approach of constraint, by explaining it to the patient, makes the connection to the machines acceptable.

Key words: Stroke; Phenomenology; Psychomotricity; Body; Constraint 


\section{Contexte}

Avec 150000 nouveaux cas par an, l'accident vasculaire cérébral (AVC) est la troisième cause de mortalité, et la première cause de handicap acquis chez l'adulte [1]. Dans la première année 30\% des patients décèdent, et seuls $60 \%$ des survivants récupèrent une indépendance fonctionnelle, tandis que $40 \%$ conservent des séquelles importantes [2]. Ainsi 225000 personnes sont classées en allocation de longue durée (ALD) pour cause d'AVC en France [3]. On distingue deux classes d'accidents vasculaires cérébraux : $80 \%$ sont ischémiques et correspondent à l'arrêt ou à la diminution de l'apport sanguin artériel vers une zone cérébrale ; $20 \%$ sont de nature hémorragique et résultent de la rupture d'un vaisseau sanguin dans le cerveau [1]. La lésion est alors focale et évolutive [4] : la zone cérébrale privée d'irrigation est rapidement entourée d'un œdème péri-lésionnel qui aggrave l'état du sujet et entraîne une altération du fonctionnement cérébral. Ainsi l'AVC se définit par l'installation brutale d'un déficit d'une fonction cérébrale ou d'un nerf crânien [4], et constitue une urgence médicale dont le premier enjeux est de stabiliser la lésion [2].

Crées en 2003, les unités neurovasculaires (UNV) offrent une optimisation des soins, une diminution de $20 \%$ de la mortalité par rapport à un service classique [4], associée à une réduction de la dépendance et de l'institutionnalisation [1], quelque soit l'âge, la gravité ou le type d'AVC [5]. Les UNV offrent une surveillance continue par une équipe multidisciplinaire formée aux risques spécifiques de l'AVC [4], pour un accueil continu, avec l'accès à un plateau technique adapté. L'UNV-A de l'hôpital Henri Mondor compte dans son équipe, une psychomotricienne présente à hauteur de 0,5 ETP auprès des patients en phase de réveil, qui participe quotidiennement aux réunions de transmissions pluridisciplinaires où sont fournies les préconisations. En France, la psychomotricité est une discipline paramédicale sanctionnée par un diplôme d'Etat [6] après trois ans de formation. Le décret de compétence du métier de psychomotricien n88-659 du 6 mai 1988 abrogé en 2004 [7], porte sur l'accomplissement d'actes de rééducation des désordres psychomoteurs (altération du schéma corporel, de la régulation tonique et tonico-émotionnelle, maladresse motrice et gestuelle) au moyen de médiations corporelles. La profession nait dès les années 50 sous l'égide d'Ajuriaguerra et de son équipe qui intègrent une perspective développementale au milieu de la psychiatrie, en abordant le corps et la motricité dans leur fonction relationnelle [8]. En rééducation, le psychomotricien accorde une importance particulière à la maîtrise corporelle dans les actes de coordination globaux ou fins [9] ; tandis que dans une optique thérapeutique, il met l'accent sur la valeur expressive et relationnelle de la motricité [9]. Ces deux approches compatibles sont souvent combinées. Dans ce service, la psychomotricienne travaille en étroite collaboration avec des kinésithérapeutes, des ergothérapeutes, des diététiciennes, une neuropsychologue, un assistant social, des infirmiers, des aides soignants et des médecins. L'équipe maintient un lien avec les aidants et les familles qu'ils renseignent sur l'évolution de leur proche hospitalisé.

De quelle manière l'AVC et les soins qui l'accompagnent modifient-ils la condition d'Etre du patient? Quelles sont les contraintes qui s'imposent à lui ? Quelle en est la réponse thérapeutique en psychomotricité ? Les vignettes cliniques présentées dans cet article concernent des prises en soin en psychomotricité au sein d'une UNV-A suite à un AVC ischémique.

\section{Modification brutale et transitoire de l'être au monde}

Le corps selon Merleau Ponty [10] est plus qu'un simple objet biologique, il constitue notre manière d'Etre au Monde: il permet à la fois de percevoir l'environnement par ses fonctions sensorielles et perceptives, et d'agir sur ce dernier par l'action motrice.

\section{Altération de la perception de soi et du monde}

En phase aigüe de l'AVC, la perception sensorielle sur laquelle s'appuie en partie la conscience corporelle [11], est altérée au niveau cérébral, tandis que s'ajoutent de nouveaux stimuli issus du contexte hospitalier.

L'exploration visuelle de l'environnement et l'auto exploration du corps sont fréquemment restreintes par des atteintes visuelles : baisse de l'acuité, amputation d'une partie du champ visuel 
(hémianopsie, quadranopsie), diplopie, paralysie oculomotrice [12]. Elles s'associent en UNV-A, à un alitement sur le dos, qui modifie l'orientation du regard vers le plafond : il est soumis à l'éclairage inhabituel des néons. Sur le plan relationnel, la communication par le regard avec les soignants se situe dans un plan vertical, tandis qu'elle se place dans un plan horizontal dans les échanges ordinaires.

Sur le plan sonore, le patient perçoit les nombreux bruits des machines environnantes (moniteur cardiaque, saturomètre), et baigne dans un bain sonore caractérisé par un langage médical technique et spécifique.

Les déficits sensitifs relèvent de l'hypoesthésie [2] soit une diminution de la sensibilité non spécifique [13], ou d'une diminution de la kinesthésie qui renseigne sur les déplacements du membre dans l'espace [14] ; s'y associent des paresthésies [2] de l'hémicorps controlatéral à la lésion sous la forme de fourmillements, de picotement ou d'engourdissement [13]. Le matériel médical comme la pose d'une voie veineuse [4] peut provoquer une sensation désagréable sur un membre sain tandis qu'un matelas anti-escarre (à air) entraine une perception des appuis inhabituelle. Le vécu corporel peut également être marqué par des douleurs neuropathiques s'apparentant à des sensations de brûlures des membres déficitaires [1], ou de l'ensemble du corps dans le cas de certaines lésions ; certains soins intrusifs tels que les prélèvements ou encore les soins d'hygiène, peuvent aussi provoquer des douleurs.

Une héminégligence de l'hémicorps et de l'espace gauche suite à une atteinte de l'hémisphère cérébral droit [13] peut s'ajouter au tableau clinique : dans ce cas, le patient ne pense plus à son côté gauche, comme s'il n'existait pas pour lui.

Les troubles sensoriels relèvent ainsi d'une sensation d'inconfort et altèrent le sentiment d'unité corporelle.

\section{Altération de l'action sur le monde}

Selon Merleau-Ponty, la motricité volontaire est un outil de connaissance de soi et d'exploration de l'environnement: "Je ne puis comprendre la fonction du corps vivant qu'en l'accomplissant moi-même et dans la mesure où je suis un corps qui se lève vers le monde. » [10]. Cette conception de la motricité fait écho au concept d'affordance de Gibson [15] : l'affordance serait une opportunité d'action ; lorsqu'un un sujet se pense capable de réaliser une action dans un contexte, il perçoit une affordance en se basant sur sa perception de l'environnement et sur la connaissance qu'il a de lui-même. Or à ce stade, le patient n'a pas encore pu réactualiser sa connaissance de ses capacités motrices tandis que sa motricité volontaire est potentiellement altérée.

Le déficit moteur est le signe le plus identifié dans l'AVC, il peut entrainer : une hémiplégie [2] caractérisée par la perte du contrôle moteur d'un hémicorps, des troubles de l'équilibre et de la coordination [13], ou encore des difficultés d'élocution [2]. A ces troubles s'ajoute la contre-indication stricte au déplacement : redresser la tête à ce stade peut avoir un effet délétère majeur voire létal ; si bien que l'accès à la position naturelle de repos si elle se fait sur le ventre ou le côté, n'est pas autorisée, ce qui va à l'encontre de l'habitus du sujet comme le défini Mauss [16]. Il arrive pourtant que des patients désorientés tentent de se lever pour aller aux toilettes : ils perçoivent une affordance erronée, car même s'ils se sentent capable de réaliser ce déplacement, ils n'ont pas conscience ni du danger encouru par leur cerveau, ni de la dégradation de leur contrôle moteur.

La satisfactions de besoins primaires comme la nutrition ou l'élimination selon les habitus du sujet n'est pas possible : les troubles de la déglutition [1] s'associent à l'interdiction de boire et de manger par sécurité ; la perte du contrôle sphinctérien [2] associée à l'injonction d'alitement nécessite parfois la mise en place d'une sonde, devant l'impossibilité d'accéder aux sanitaires. Si Merleau-Ponty évoque la possibilité de «dilater» notre Etre au monde pour incorporer un instrument [10](ici la sonde) ce processus n'est pas rapide et relève plutôt d'une habitude, impliquant un accord entre le 
vécu du patient et ce qui est visé avec l'outil : un défaut de compréhension au sens de Merleau-Ponty pousse certains patients à arracher les sondes.

Les contraintes médicales, associées aux troubles moteurs, restreignent la réactualisation du schéma corporel par l'action. Durant la phase aigüe de l'AVC, le sujet n'a plus l'opportunité d'orienter sa motricité vers l'environnement ou simplement vers ses propres besoins physiologiques. L'expérience du corps vivant est désorganisée et sous la dépendance temporaire d'un tiers.

\section{Altération du sentiment d'identité et des facultés relationnelles}

La diminution physique et intellectuelle subie par le patient à ce stade, génère une forte angoisse [2] et peut s'accompagner de troubles cognitifs majeurs [1] qui déstabilisent le patient. A son arrivée en UNV-A, il est brutalement dépossédé de son identité : ses papiers et ses vêtements lui sont retirés. Ses facultés d'être communiquant sont diminuées par les troubles neurologiques [17]. L'aphasie fluente (anciennement Wernicke) entraine une altération de la compréhension ou du décodage, les représentations des formes auditives ou visuelles des mots ne sont plus associées à leur représentation sémantique [18] : le patient ne comprend plus ou peu ce qui lui est dit, et son langage est déformé voire incompréhensible (néologismes) sans pour autant qu'il en ait conscience. A l'inverse une aphasie non fluente (anciennement Broca) souvent associée à une hémiplégie, se caractérise par un appauvrissement massif du langage pouvant aller jusqu'au mutisme, bien que la compréhension soit préservée [18]: les facultés de commande et les coordinations motrices des muscles faciaux sont préservées mais le patient ne peut pas élaborer de phrase (ni écrire), tout en ayant conscience de son trouble. Pour comprendre le patient dans sa réalité psychique, les professionnels s'appuient sur des indices corporels et les éléments de langages [19], bien qu'ils soient ici modifiés.

La faculté d'Etre à l'autre est ainsi altérée dans sa dimension identitaire et communicante.

\section{Corps augmenté}

La surveillance des fonctions vitales est une nécessité et de nombreux instruments de mesure sont mis en place : le corps vivant du patient est quantifié, numérisé. Ces outils permettent un accès au corps vivant, ou viv@ant [20] et deviennent les nouveaux organes de communication avec les soignants. De nombreuses mesures des constantes sont ainsi instaurées [4] : le monitoring de la pression artérielle, du rythme cardiaque, de la saturation en oxygène et une mesure de la température ; l'électro cardiogramme, la prise de la glycémie, la pause de voie veineuse ainsi que des prélèvements biologiques sont également réalisés. Le passage par la machine I.R.M. (ou scanner s'il existe une contre-indication) est systématique, pour une durée variable, afin de déterminer la nature, l'étendue et la localisation de la lésion [4]. Le patient est donc soumis à un accordage aux machines selon plusieurs modes : non invasif restrictif, avec le placement des capteurs contre la surface cutanée limitant les mouvements; invasif avec les poses de voies veineuses et autres prises de sang (analysées ultérieurement). Le patient est ainsi contraint d'intégrer ce nouveau câblage à son schéma corporel selon un processus d'incorporation [10]. L'organisation spatiale des chambres est la suivante : les écrans de monitoring sont situés à la tête du lit, ou sur le coté mais pas dans le champs visuel (déjà réduit) du patient. Cet accès aux données du corps vivant n'est alors pas perceptible par la lecture, et ne va pas actualiser la connaissance du corps vécu comme dans l'utilisation classique du quantifiedself [20] où d'ordinaire l'utilisateur visualise ses chiffres. En revanche, il existe un retour perceptif des machines sur le canal auditif : les bruits varient en cas d'anomalie. Il est complexe de savoir dans quelle mesure ce retour sonore entraine une actualisation du corps vécu chez le patient.

\section{Pratique psychomotrice}

\section{$\hat{E}$ tre au monde : situer le patient dans son environnement}

Après s'être présentée au patient, la psychomotricienne lui fournit des informations sur l'environnement et le contexte dans lequel il évolue : où il se trouve, ce qui lui arrive. Elle décrit 
sommairement l'organisation de la pièce : où se situe la porte et les machines qu'il entend et leur intérêt. La contrainte d'alitement est présentée comme essentielle à la survie dans l'attente des résultats médicaux, cependant il convient d'insister sur le caractère transitoire de cette injonction. Il s'agit d'aider le patient à se situer en lui fournissant les informations qu'il ne peut pas percevoir et en donnant un sens à celles qu'il perçoit.

\section{Être à soi : réactualiser le schéma corporel}

Afin d'aider le sujet dans la réappropriation de son corps, la psychomotricienne s'appuie sur la sensori-motricité. Elle propose des touchers thérapeutiques, des mobilisations passives ou des mouvements accompagnés (participation active partielle du patient) des membres, étayés par un guidage verbal de l'attention vers les parties du corps.

Il est fréquent que les patients hémiplégiques ou hémi-négligents laissent un de leur bras pendre dans le vide dans une position défavorable à la circulation sanguine, de sorte que le bras gonfle. La psychomotricienne vient alors replacer le bras dans une position adaptée, le contact physique est précédé d'une mise en mots : «Je vais poser ma main sur votre bras, là il est gonflé, je vais vous aider à le remettre dans une position confortable. » Il s'agit de mobiliser l'attention du patient vers son membre avec l'idée d'en prendre soin.

La psychomotricienne accompagne la réactualisation du schéma corporel par le biais de la sensorialité lors de mouvements en contact. Lors de mouvements accompagnés, elle réalise des mobilisations passives d'un membre, couplés à des inductions verbales orientant l'attention (et la vue) du patient vers la partie mobilisée. Les stimuli provoqués relèvent de sensations proprioceptives par stimulation tendineuse et des fuseaux neuromusculaires, tactiles et thermiques par le contact entre les mains du rééducateur et la peau du patient. Ce dernier est sollicité pour regarder ses propres membres au moment de la mobilisation, pour une intégration optimale de la perception. Des auto-contacts sont proposés, jouant ainsi sur la dimension touchant-touché du corps à travers sa condition double d'objet percevant-perçu comme le présente Merleau-Ponty. Les mouvements sont réalisés sur des schèmes d'ouverture-fermeture, dans les différents plans de l'espace: il s'agit également de reprendre possession de l'espace proche.

De manière active, il est demandé au patient d'activer des groupements musculaires du membre que tient la psychomotricienne. La perception de la professionnelle est alors mise en relation avec l'action du patient : «Poussez sur ma main. Là, je ne sens pas grand-chose. Essayez encore ». Il s'agit ici d'activer la commande motrice même si le résultat effectif n'est pas aussi performant que l'attendu du patient. La même consigne est proposée du coté sain afin d'entrainer une comparaison de force musculaire par le sujet lui-même au regard de la commande consciente qu'il a du mouvement.

La psychomotricienne propose ainsi des stimulations sensorimotrices avec un objectif de prise de conscience corporelle, décliné sur des modes actifs et passifs, basés sur une interaction corporelle dynamique.

\section{Être à l'autre : corps en relation}

Les exercices proposés par la psychomotricienne impliquent une dynamique relationnelle à travers le dialogue tonique. La psychomotricienne adapte sa communication corporelle : elle modifie sa posture et son positionnement dans l'espace selon l'installation du patient. Ainsi elle s'oriente de manière à établir un contact visuel satisfaisant dans la partie fonctionnelle du champs visuel du patient, en diminuant autant que possible la verticalité du rapport visuel vers une horizontalité plus proche de l'habitus de communication.

Le langage de la psychomotricienne est adapté à l'état du patient (bien qu'il soit parfois impossible de déterminer le degré de compréhension et de conscience). Le vocabulaire employé est ainsi simplifié et la longueur des phrases est raccourcie pour expliquer au patient. 
Un autre enjeu consiste à rendre acceptable le ralliement du corps au machine, parfois rejeté avec violence: il n'est pas rare de voir les patients arracher les câbles et perfusions. Afin d'accompagner cette extension artificielle du schéma corporel, la psychomotricienne prend le temps d'expliquer au patient le rôle des différents capteurs : la machine fait le lien à distance avec les infirmiers, qui reçoivent l'ensemble des données et des constantes sur leurs écrans. Ainsi les capteurs permettent la mise en relation du sujet avec les soignants, dans le but d'assurer sa survie et sa santé : la machine devient alors un organe artificiel de communication. Le patient informé redevient ainsi acteur-émetteur dans la relation par le biais des données de son corps vivant. La contrainte subie dans le vécu, se transforme en action de communication. L'information et la compréhension des mécanismes comblent alors l'absence de feedback perceptif, et rend acceptable un dispositif contraignant, allant parfois jusqu'à le transformer dans le vécu en dispositif accepté et utile. Cependant, la confusion et les difficultés mnésiques peuvent nécessiter de nombreux rappels.

\section{Discussion : l'émersion en psychomotricité}

Dans l'approche phénoménologique, sentir et se mouvoir [21] s'associent pour constituer la condition de l'Etre au monde, comme mode d'existence du sujet. En UNV-A, le sujet se voit diminué dans ces deux aspects par l'AVC et les contraintes médicales qui accompagnent les premiers soins. La démarche des soins en psychomotricité s'appuie sur deux dimensions du corps phénoménologique : le corps vivant et le corps vécu.

\section{Actualiser le corps vivant}

Le corps vivant est un objet physique biologique qui s'actualise par l'activation des réseaux sensorimoteurs du schéma corporel ; il est influencé par le contexte social à travers les techniques du corps (habitus) [16], ces «gestes et postures incorporés [inhérents à la culture] que nous accomplissons sans nous en rendre compte » [22]. Il est l'un des deux composants essentiels des affordances, définies comme les possibilités d'action d'un individu sur l'environnement au regard de ses capacités sensorimotrices [15]. Par immersion dans le monde, le corps vivant s'actualise continuellement [23], modifiant ainsi ses qualités internes par un phénomène de plasticité [22]. Les séquelles de l'AVC impactent directement le corps vivant dans ses fonctions sensorimotrices tandis que les contraintes médicales restreignent l'accès aux habitus. Lorsque la psychomotricienne demande au sujet de réaliser une action motrice (pousser ou serrer la main), elle lui demande d'activer son corps capacitaire [24] qui entraine une actualisation du schéma corporel au regard du contexte spatiotemporel actuel.

L'action motrice actualise la connaissance des capacités dans le vécu corporel. La capacité se définit ainsi comme la conscience du corps vécu, de ce qu'un sujet croit possible de faire de son corps [23]. Cependant en l'absence d'actualisation, le sujet peut percevoir des potentialités d'action (affordances) [15] en inadéquation avec ses possibilités actuelles : c'est le cas des patients qui veulent se lever précocement alors qu'ils risquent de chuter, ce dont ils n'ont pas conscience. L'action permet de réactualiser la perception des affordances pour certains segments du corps dans un référentiel spatio-temporel, ce travail sera poursuit lors de la phase de rééducation qui suit les soins aigus.

\section{Actualiser le corps vécu}

Le corps vécu [23] se construit à partir des phénomènes de l'activité du corps vivant qui sont accessibles à la conscience et teintés de représentations : il suppose une conscience corporelle et une conscience de soi, dans une vision subjective en première personne. Cette image est moins intense que le schéma corporel, car toutes les sensations du corps vivant ne parviennent pas à la conscience du corps vécu [22]. La conscience corporelle (bodily self consciousness) repose sur la faculté à sentir son corps ou ses parties comme siennes (body ownership) et à s'identifier à son corps (self indentification), tout en étant capable de le situer dans l'environnement (self location) [25]. En phase aigüe de l'AVC 
la fatigue et les difficultés attentionnelles [12] et cognitives marquées associées à la confusion [1] désorientent et entravent cette conscience corporelle essentielle à l'actualisation du corps vécu.

L'émersion est le processus inconscient selon lequel certaines données de l'activité du corps vivant parviennent plus ou moins déformés à la conscience du corps vécu [22], elle peut être consciemment provoquée par les techniques d'awareness [22]. La stimulation sensorimotrice effectuée par la psychomotricienne s'inscrit dans cette optique : à travers un accompagnement verbal, une attention dirigée vers les sensations, et une orientation du regard vers le membre en mouvement. La mise en mot place le patient dans son expérience en première personne : " votre main gauche touche votre joue » apporte le sens et le contexte utile à l'appropriation de l'expérience. Le caractère actif, même partiel lorsque le mouvement doit être aidé, permet au patient d'être acteur de l'action ce qui renforce le sens de la «possession de son corps » [26].

L'action motrice actualise ainsi la connaissance des capacités dans le vécu corporel. La capacité se définit comme la conscience du corps vécu, de ce qu'un sujet croit possible de faire de son corps [23], elle est intimement liée à la perception des affordances. Cependant en l'absence d'actualisation, le sujet peut percevoir des potentialités d'action [15] en inadéquation avec ses possibilités actuelles : c'est le cas des patients qui veulent se lever précocement alors qu'ils risquent de chuter, ce dont ils n'ont pas conscience. L'action permet de réactualiser la perception des affordances pour certains segments du corps dans un référentiel spatio-temporel, ce travail sera poursuit lors de la phase de rééducation qui suit les soins aigus.

\section{La relation comme support émersif}

La perception globale et unifiée du corps vivant repose sur le traitement d'informations somatosensorielles et spatiales, mais aussi relationnelles [27] ; la psychomotricienne par son dialogue tonique et sa mise en mots devient support de ce mécanisme émersif. Rétablir la dimension relationnelle du corps est un enjeu majeur dans l'approche psychomotrice : Ajuriaguerra déclare en 1983 à l'Université de Barcelone que « Notre corps n'est rien sans le corps de l'autre ». Il défend ainsi un ancrage corporel et interactif [28], encore partagé par les psychomotriciens aujourd'hui. Ici la psychomotricienne agit au contact du patient en partageant avec lui ce qu'elle perçoit de son corps. Le mode de relation reste cependant différent du contact classique car il est à l'initiative du professionnel : dans le contexte de soins, le patient n'est pas à l'initiative de l'échange et voit son rythme dépendre des la disponibilités et des sollicitations des soignants. La relation partiellement entravée dans les troubles de la communication, nécessite donc une attention particulière du soignant face aux réactions du patient. Si l'AVC et son contexte peuvent entrainer un état de stress posttraumatque [29], les désaccordages entre patient et soignants sont également des situations à risque [30] qui peuvent entrainer des difficultés ultérieures dans le parcours de soin.

\section{Conclusion}

Les principales contraintes médicales en UNV-A, indispensables à la survie du patient, reposent sur l'alitement strict dorsal à plat, et le raccordement aux machines. Elles freinent les possibilités de réactualisation d'un schéma corporel déjà altéré par les séquelles de l'AVC. L'approche psychomotrice s'oriente ainsi vers une réappropriation corporelle dynamique, émersive et située.

\section{Déclaration d'intérêt}

Les auteurs déclarent ne pas avoir de conflits d'intérêt en rapport avec cet article.

\section{Références}

[1] Gosseaume A, Lejeune P, De Marco O, Urbanczyk C. Mise au point sur les accidents vasculaires cérébraux. Rev Francoph Orthopt 2016;9:71-6.

[2] Bezanson C. Les accidents vasculaires cérébraux. Rev Francoph Orthopt 2016;9:63-7.

[3] Zuber M. Le Plan AVC 2010-2014. Prat Neurol - FMC 2011;2:3-5. 
[4] Goulmy M. Prise en charge de l'accident vasculaire cérébral à sa phase aiguë. Rev Francoph Orthopt 2016;9:124-7.

[5] Debiais S, Bonnaud I, Giraudeau B, Perrotin D, Gigot J-L, Saudeau D, et al. Création d'une filière neuro-vasculaire régionale : évaluation de la prise en charge à 18 mois. Rev Neurol (Paris) 2007;163:817-22.

[6] Boutinaud J, Rodriguez M, Moyano O, Joly F. Les thérapeutiques psychomotrices aujourd'hui : perspective dialectique et approche intégrative. Neuropsychiatr Enfance Adolesc 2013;61:250-8.

[7] Décret n88-659 du 6 mai 1988 relatif à l'accomplissement de certains actes de rééducation psychomotrice. 1988.

[8] Rodriguez M. Médiation corporelles en psychomotricité : évolution des idées. Où En Est Psychomot. Etat Lieux Perspect., Paris: IN PRESS; 2014, p. 37-42.

[9] Potel C. Cinq axes essentiels de l'intervention en psychomotricité. Etre Psychomot. Un Métier Présent Un Métier Avenir, Paris: Erès; 2010, p. 309-20.

[10] Merleau-Ponty M. Phénoménologie de la perception. Paris: Gallimard; 1976.

[11] Salomon R, Noel J-P, Łukowska M, Faivre N, Metzinger T, Serino A, et al. Unconscious integration of multisensory bodily inputs in the peripersonal space shapes bodily selfconsciousness. Cognition 2017;166:174-83.

[12] Bouly de Lesdain A. Orthoptie en phase aigüe d'un Accident Vasculaire Cérébral. Rev Francoph Orthopt 2016;9:77-81.

[13] Rohkamm R, Grosshans B. Atlas de poche de neurologie. 2e édition. Paris: Médecine Sciences Publications; 2016.

[14] Le Cavorzin P. Neurophysiologie de la fonction proprioceptive et récupération postlésionnelle. Kinésithérapie Rev 2012;12:7-14.

[15] Luyat M, Regia-Corte T. Les affordances : de James Jerome Gibson aux formalisations récentes du concept. L'Année Psychol 2009;Vol. 109:297-332.

[16] Mauss M. Les techniques du corps. J Psychol 1936;32:271-293.

[17] Capron J. Retentissement psychiatrique de l'AVC. NPG Neurol - Psychiatr - Gériatrie 2015; 15:353-8.

[18] Gil R. Neuropsychologie. Elsevier Health Sciences; 2018.

[19] Ciccone A. Chapitre 1 - Violences des requêtes sociales. Violence Dans Soin, n.d., p. 19-46.

[20] Andrieu B. L'osmose technique avec son corps viv@ nt: une auto-santé connectée du patient immersif. LÉvolution Psychiatr 2016;81:253-265.

[21] Robinson B. Corps et psychomotricité. Paris: Editions L'Harmattan; 2014.

[22] Andrieu B. Sentir son corps vivant: Tome 1, Emersiologie. Librairie Philosophique Vrin; 2016.

[23] Andrieu B. Le corps capacitaire : Une performativité du vivant. Presses universitaires de Paris Ouest; 2018.

[24] Nobrega TPD, Schirrer M, Legendre A, Andrieu B. Sentir le vivant de son corps : trois degrés d'éveil de la conscience. Staps 2017:39-57.

[25] Blanke O, Slater M, Serino A. Behavioral, Neural, and Computational Principles of Bodily SelfConsciousness. Neuron 2015;88:145-66.

[26] Jeannerod M. De l'image du corps à l'image de soi, From the body image to the sense of self. Rev Neuropsychol 2012;me 2:185-94.

[27] Dieguez S, Lopez C. The bodily self: Insights from clinical and experimental research. Ann Phys Rehabil Med 2017;60:198-207.

[28] Rodriguez M. De la place du corps dans les thérapeutiques psychomotrices. J Psychol 2012:225.

[29] Frénisy M-C, Minot D, Soutenet M, Amiot N. Accidents vasculaires cérébraux : approche psychopathologique et approche neuropsychologique. À propos d'un cas : M. J. Ann MédPsychol Rev Psychiatr 2005;163:65-72.

[30] Paillard A. Chapitre 10 - Désaccordages, échecs, violences dans la rencontre patient-soignant. Violence Dans Soin, n.d., p. 199-216. 